\title{
Nalmefene to prevent Epidural morphine-induced pruritus after Caesarean delivery: a randomized, placebo-controlled clinical trail
}

Chaochao Zhong ( $\square$ zhong249767626@163.com )

Affiliated Hospital of Nantong University

\section{Xingguo $\mathrm{Xu}$}

Affiliated Hospital of Nantong University

\section{Xiang Zhu}

Affiliated Hospital of Nantong University

\section{Yibin Qin}

Affiliated Hospital of Nantong University

\section{Haihang Miao}

Affiliated Hospital of Nantong University

Xinchong Huang

Affiliated Hospital of Nantong University

\section{Su Cao}

Affiliated Hospital of Nantong University

\section{Research article}

Keywords: Nalmefene, Epidural morphine, Pruritus, Caesarean delivery

Posted Date: November 26th, 2019

DOI: https://doi.org/10.21203/rs.2.17436/v1

License: (c) (i) This work is licensed under a Creative Commons Attribution 4.0 International License. Read Full License 


\section{Abstract}

Background. Epidural morphine provides an effective analgesic effect on cesarean section patients, while epidural morphine-induced pruritus is a very common side-effect that is difficult to prevent or treat. The aim of this study was to determine whether a $\mu$-opioid antagonist with central and peripheral effects could reduce morphine-induced pruritus.

Methods. Eighty patients who underwent elective cesarean delivery under spinal aesthesia with epidural morphine $3 \mathrm{mg}$ were divided into two groups of 40 each in this prospective, randomized study. After delivery, participants received either intravenous nalmefene hydrochloride $50 \mu \mathrm{g}$ ( Nalmefene group, $\mathrm{n}=40$ ) or saline(Placebo group, $n=40$ ). Pruritus, nausea, pain, and side-effects were assessed at 2, 4, 8, 12, 24h after epidural morphine administration.

Results. Eighty women completed the study. Compared to IV saline, the total incidence of pruritus within the first 24 hours was reduced in IV nalmefene group (37.5\% vs 65\%, P=0.003). IV nalmefene also reduced the severity of pruritus. There was no significant difference in the incidence of nausea and vomiting. Remarkably, although the pain scores were significantly higher at 8,12 and $24 \mathrm{~h}(\mathrm{all} \mathrm{P}<0.05)$ in the nalmefene groups, but there was no difference between two groups in the number of patients who needed analgesic treatment $(P=0.37)$.

Conclusions. A single dose of IV nalmefene $50 \mu \mathrm{g}$ can reduce the overall severity and incidence of epidural morphine-related pruritus without adversely affecting the quality of postoperative analgesia. TRIAL REGISTRY: ChiCTR1900022268. Registered on 2 April 2019

\section{Background}

One of the most common side effect of epidural morphine when used for postoperative analgesia after cesarean section is pruritus, it has been reported in $30-100 \%$ of patients ${ }^{1}$. Pruritus is an unpleasant sensation that causes the reflex to stratch, even though it has a significant effect on the patient's health², it was still not abandoned clinically for its excellent postoperative analgesia after caesarean delivery. ${ }^{1}$

In fact, many medications have been studied to prevent epidural opioid-associated pruritus, morphineinduced pruritus showed no or weak response to antihistamines, serotonin antagonists, and propofol ${ }^{3,4}$. Several other drugs with different effects and side effects have been studied, including gabapentin, nalbuphine and pentazocine. Currently, the exact mechanism of neuraxial opioid-induced pruritus remains unknown, it is generally believed that it is mediated by $\mu$ receptors, because opioid receptors has rich expression in the central nervous system. ${ }^{5}$ Therefore, the most effective drug for the treatment of pruritus caused by epidural morphine is an $\mu$-opioid antagonists, such as naloxone, but these acting $\mu$ opioid antagonists have the potential to adversely affect analgesia ${ }^{3}$.

There is experimental evidence suggesting that naloxone alleviate the itching of animals caused by opioids ${ }^{6}$, but naloxone can also antagonize the analgesic effect while relieving itching. Nalmefene is a 
derivative of naloxone, an opioid antagonist which has higher affinity with $\mu$ receptor and long-lasting effect. Studies have shown that low doses of nalmefene has no effect on analgesia or postoperative pain ${ }^{7}$. At present, there are few studies focused on pruritus with nalmefene, thus we hypothesized that IV nalmefene would significantly reduce the severity and incidence of pruritus induced by epidural morphine after caesarean section.

\section{Methods}

After obtaining the approval of ethics committee and written informed consent. The study was registered with Chinese Clinical Trial Registry number: ChiCTR1900022268, which was registered in 2 April 2019, http://www.chictr.org.cn/edit.aspx. The work has been reported in line with Consolidated Standards of Reporting Trials (CONSORT) Guidelines.

80 patients undergoing elective cesarean section were recruited. The study was a randomized, placebocontrolled trial that was conducted from April to June 2019.

Inclusion criteria included ASA class $\$ or $\$ women, aged $18 \mathrm{yr}$ or more undergoing elective caesarean section under epidural anasthesia including epidural morphine. Exclusion criteria were as follows: no consent to study, coagulation dieases, liver or kidneyfailure, preexisting pruritus, previous surgery in the lumbar spine.

Ater arrival in the operating room, all patients received epidural anesthesia. Epidural catheterization was performed using an 18-gauge epidural needle and a epidural catheter at the T12-L1 or L1-L2 level. 0.5\% ropicacaine $12 \mathrm{ml}(60 \mathrm{mg})$ and morphine $(3 \mathrm{mg})$ were administered. Patients were placed in the supine position, and intraoperatively, systolic blood pressure was maintained above $100 \mathrm{mmHg}$ by colloid liquid and intermittent injection of phenylephrine.

The patients were divided randomly into two groups: a placebo group (saline injection) and a nalmefene group(50ug nalmefene injection). The nalmefene or placebo was administered near the end of surgery, and other intraoperative anaesthetic and postoperative care were standardized in this study. Baseline date such as patient age, characteristics, operation time were collected. The incidence of intraoperative pain or nausea/vomiting was recorded. In the postoperative period, the level of pruritus, pain score, and other adverse effects included nausea and vomiting were evaluated $2,4,8,12$, and $24 \mathrm{~h}$ after nalmefene or saline administration. 24 hours after delivery, patients were reexamined to determine the total incidence of pruritus, whether treatment for pruritus or pain had been required. The severity of pruritus was assessed by verbal rating scale: $0=$ no pruritus, $1=$ mild pruritus, $2=$ moderate pruritus, $3=$ severe pruritus. ${ }^{3}$ Pain scores with VAS, range 0 and 10 , with $0=$ no pain and $10=$ unbearable pain. Intravenous ondansteron $(4 \mathrm{mg})$ in the treatment of nausea and vomiting. When necessary, non-steroidal antiinflmmatory drugs or intravenous morphine were used to assist postoperative analgesia.

Statistical analysis 
Statistical analysis was performed by use of the statistical software SPSS 13.0. Power Analysis and Sample Size (PASS) software (NCSS), LLC, Kaysville, UT, USA) was used for the sample size calculation. Clinically, it is considered which has therapeutic significance to reduce the incidence of pruritus by $30 \%$. The sample size was estimated with $a=0.05$ and $\beta=0.95$, respectively. In the preliminary study, the incidence of pruritus in the control group was $65 \%$. Therefore, each group must include at least 32 patients for the requirement of $30 \%$ reduction of pruritus incidence.

After normality testing using Levene method, the patients'data will be presented as the mean and standard deviation (SD) if they are normally distributed; otherwise, they will be presented as the mean and interquartile range. count data groups were analyzed using chi-square test. The $\chi 2$ test and Fisher's exact test will be used for compare proportional data, the Nemenyi test will be used to compare the

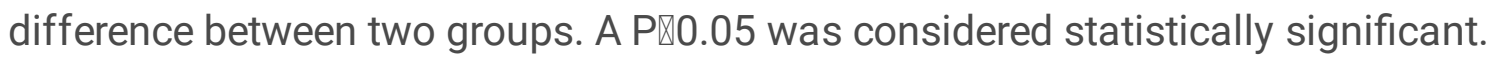

\section{Results}

Among the 84 patients enrolled, 4 were excluded. In total, 80 patients were recruited in this study (placebo group $n=40$, nalmefene group $n=40$ ). There were no differences in baseline characteristics between the two groups (Table 1).

The primary outcome measures of incidence of pruritus was $32.5 \%(13 / 40)$ in the nalmefene group and $65 \%(26 / 40)$ in the placebo group (Table 4$)$. There was a significant difference between the two groups $(P<0.05)$. Table 2 shows the severity of pruritus with significant differences in the percentage and treatment of moderate or severe pruritus. In the nalmefene group, 4 patients required treatment of pruritus (ondansetron or antihistamine) while 19 patients required treatment in placebo group for pruritus (Table $4, \mathrm{P}<0.01$ ). The level of pruritus was significantly different between the two groups after $2,4,8$, and $12 \mathrm{~h}$ ( Table $2, \mathrm{P}<0.05$ ).

Expect for the 8, and $12 \mathrm{~h}$, VAS scores were similar between the two groups (Table 3). Although the VAS scores were sightly higher in nalmefene group patients at 8 , and $12 \mathrm{~h}$, but there was no significantly different between two groups about the number of patients required treatment for pain( Table $4,10 \%$ vs $15 \%, p=0.37$ ).

The incidence of nsusea in the first $24 \mathrm{~h}$ was $17.5 \%$ and $25 \%$ in the placebo and nalmefene groups, respectively $(P=0.29)$. while the incidence of vomiting was $7.5 \%$ vs $10 \%$ in two groups $(P=0.51)$. There were no differences in terms of postoperative nausea and vomiting. 
Figure 1 Flow chart of the study

Table 1 Patient characteristics, obstetric, and baseline clinical data. There were no significant differences between groups.

\begin{tabular}{lcc}
\hline \multicolumn{2}{c}{ Placebo $(\mathrm{n}=40)$} & Nalmefene $(\mathrm{n}=40)$ \\
\hline Age $(\mathrm{y})$ & $27.5 \pm 3.7$ & $26.4 \pm 3.6$ \\
Weight $(\mathrm{kg})$ & $73.6 \pm 4.1$ & $73.4 \pm 4.6$ \\
BMI $(\mathrm{kg} / \mathrm{m} 2)$ & $29.9 \pm 2.7$ & $29.9 \pm 2.8$ \\
Nulliparous & $25(62.5 \%)$ & $23(57.5 \%)$ \\
Op time (min) & $46.6 \pm 4.6$ & $45.5 \pm 4.2$ \\
\hline
\end{tabular}

Values are expressed by mean $\pm \mathrm{SD} \llbracket$ standard deviation\except nulliparous represent as $(\mathrm{N}$, percent\%).

Table 2 the severity of pruritus at 2,4,8,12,and $24 \mathrm{~h}$ after epidural injection of morphine was assessed, Categorical variables were presented as counts(and percentage). After $2,4,8$, and $12 \mathrm{~h}$, there was significant difference in the degree of pruritus between the two groups $\left({ }^{*} \mathrm{P}<0.05\right)$.

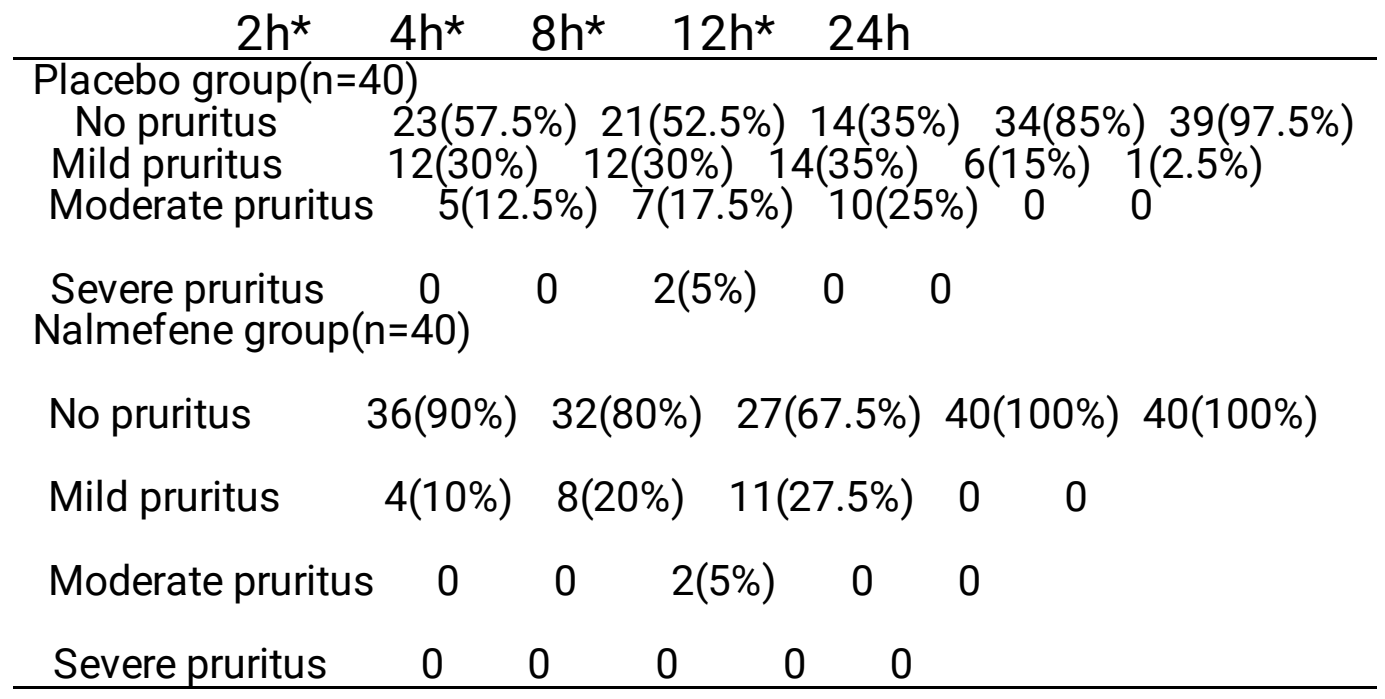


Table 3 Pain VAS scores, values are expressed by mean \pm SD (standard deviation). the VAS scores in nalmefene group were higher than placebo group at 8 , and $12 \mathrm{~h}\left({ }^{*} \mathrm{P}<\right.$ $0.05)$.

\begin{tabular}{ccccccc}
\hline $2 \mathrm{~h}$ & $4 \mathrm{~h}$ & $8 \mathrm{~h}^{\star}$ & $12 \mathrm{~h}^{\star}$ & $24 \mathrm{~h}$ & & \\
\hline Placebo group & $1.2 \pm 0.8$ & $1.9 \pm 0.8$ & $2.1 \pm 0.9$ & $2.2 \pm 0.7$ & $2.0 \pm 0.8$ \\
Nalmefene group & $1.2 \pm 0.7$ & $1.9 \pm 1.1$ & $2.8 \pm 0.8$ & $3.1 \pm 0.7$ & $2.9 \pm 0.8$
\end{tabular}

\section{Table 4 Adverse events}

\begin{tabular}{|c|c|c|c|c|}
\hline \multirow{2}{*}{\multicolumn{2}{|c|}{ Placebo $(n=40)$}} & \multicolumn{2}{|c|}{ Nalmefene $(n=40)$} & $\mathrm{P}$ \\
\hline & 7 & 10 & 0.29 & \\
\hline Vomitting(n) & 3 & 4 & 0.51 & \\
\hline Pruritus(n) & 26 & 13 & $<0.05$ & \\
\hline treatment of & $\operatorname{tus}(\mathrm{n}) 1 \mathrm{c}$ & 4 & & $<0.01$ \\
\hline reatment o & (n) & 6 & & 37 \\
\hline
\end{tabular}

\section{Discussion}

In this prospective, randomized, placebo-controlled clinical trail we compared the antipruritic effect of nalmefene and normal saline in patients undergoing cesarean delivery. Our results show that a single dose of IV nalmefene 50 $\mu$ g can reduce the overall severity and incidence of epidural morphine-related pruritus without adversely affecting the quality of postoperative analgesia.

Pregnant women are more prone to itching, for whom the incidence is dose dependent ${ }^{8,9}$ and located on the face, neck, and upper thorax ${ }^{10}$. Although the exact mechanisms of pruritus is not clear, a lot of progress has been made recently in identifying signal molecules as potential targets of anti-pruritus therapies ${ }^{11,12}$. At present, we believed that opioid induced pruritus may by mediated by the interaction with $\mu$-opioid receptors, because $\mu$-receptor antagonists can effectively reduce pruritus in patients with pruritus by acting on central and possibly peripheral targets ${ }^{13}$. As we expand our understanding, we also 
have the ability to set more specific targets for novel treatments, which will hopefully improved efficacy and reduce adverse effects. Currently, the most effective treatment of epidural morphine-induced pruritus is an $\mu$ receptor antagonist, such as naloxone. However, the dosage of naloxone for pruritus treatment is limited to a small dosage because high dosage of naloxone can reverse the analgesic effect of opioids ${ }^{14}$. nalmefene is a novel $\mu$-opioid antagonist derivative of naloxone, it has stronger affinity with $\mu$-opioid compared with naloxone. Secondly, both naloxone and nalmefene can readily crossing the blood-brain barrier ${ }^{15}$, while naloxone metabolized faster in body. Importantly, Clinical dose of nalmefene seems to antagonize the side effects of opioids, but has little effect on pain.

This clinical study was to observe the effect of nalmefene on pruritus caused by epidural morphine. The incidence of pruritus was reduced to $32.5 \%$ in the nalmefene group. Although the percent of pruritus still higher than some studies have reported ${ }^{16,17}$, it might be related to the dosage of morphine. Meanwhile, this result might be due to the improper dosage and administration time, or the fact that nalmefene cannot affect other itching transmitters, for example prostaglandins ${ }^{18}$, the neurotransmitters glutamate and $\mathrm{GABA}^{19}$ or NMDA receptors ${ }^{20}$, all of which have important role in pruritus.

In our observations, VAS scores of the two groups were similar except at 8 and $12 \mathrm{~h}$ after operation. The VAS scores were sightly higher in nalmefene group at 8 and $12 \mathrm{~h}$, it seemed that nalmefene was reduced pruritus and impaired the analgesic efficacy of epidural morphine. Surprisingly, there was no difference between two groups in the number of patients who needed analgesic treatment. That meant nelmefene can reduce the overall severity and incidence of epidural morphine-related pruritus without adversely affecting the quality of postoperative analgesia. In addition to causing itching, epidural morphine also induce nausea and vomiting by activating serotonin receptors in the trigger area of the vomiting chemoreceptor $^{21}$. But, there was no significant difference in nausea and vomiting between the two groups.

There are some limitations to our study. First, the trial only studied the effect of a single dose of nalmefene after the administration of epidural morphine in women undergoing Caesarean section. If we change the dosage of nalmefene or the type of patient, the results may be different. Secondly, we did not study the dose-dependence effect of nalmefene on pruritus; so we did not optimize the dose. In the future, further research is needed to explore whether the treatment of pruritus by nalmefene is dose-dependent, whether it also has therapeutic effect on pruritus caused by other opioids epidural administered, and quantify an optimal dose can meet the requirements of antipruritic effect and reduce side effects to the greatest extent.

\section{Conclusions}

In conclusion, A single dose of IV nalmefene $50 \mu \mathrm{g}$ can reduce the overall severity and incidence of epidural morphine-related pruritus without adversely affecting the quality of postoperative analgesia.

\section{Abbreviations}


ASA: American society of anesthesiology; VAS: visual analog scale

PASS: Power Analysis and Sample Size

\section{Notes}

Ethics approval and consent to participate

This study was obtained ethics committee of Affiliated Hospital of Nantong University approval (approved number: 2018-K067), each patient provided a written informed consent.

Consent for publication

We transfer the ownership of copyright to the journal "BMC anesthesiology" should our work be published in this journal. We declare that the article is original. We have participated in drafting and revising the manuscript submitted, whose contents they approve.

Availability of data and materials

All necessary data supporting our findings has been presented within the manuscript. The datasets used and/or analyzed during the current study are available from the corresponding author on reasonable request.

Competing interests

The authors declare that we have no competing interests.

Funding

We declare that this clinical trial is funded by Nantong City Science and Technology Bureau and completed by Nantong University Hospital.

Authors' contributions

CCZ carried out the studies, and drafted the manuscript. XGXロXZ and HHM performed the statistical analysis and helped to collect the data. YBQ and SC helped to revise the manuscript. All authors read and approved the final manuscript.

Acknowlegements

The authors are grateful to professor Su Cao, Department of Anesthesiology, Affiliated Hospital of Nantong University, for her help in the design and implementation of this project.

Statement

The study adheres to CONSORT guidelines. 
Authors' Information

Chaochao Zhong, Email: zhong249767626@163.com

Xingguo Xu, Email: TDFYXXG@126.com

Xiang Zhu, Email: Bobofly8850@sina.com

YiBin Qin, Email: 572537172@qq.com

Haihang Miao, Email: miaohaihang@163.com

Xinchong Huang, Email: hxcnt115@sohu.com

Su Cao, Email: mzkcs@sina.com

\section{References}

1. Kumar K, Singh SI. Neuraxial opioid-induced pruritus: an update. J Anaesthesiol Clin Pharmacol 2013;29:303-7.

2. Yosipovitch G, Carstens E, McGlone F. Chronic itch and chronic pain: analogous mechanisms. Pain 2007;131:4-7.

3. Lockington PF, Fa'aea P. Subcutaneous naloxone for the prevention of epidural morphine induced pruritus in elective caesarean delivery. Anesthesia 2007;62:672-676.

4. Bonnet MP, Marret E, Josserand J. Effect of prophylactic 5-HT3 receptor antagonists on pruritus induced by neuraxial opioids: a quantitative systematic review. Br J Anaesth 2008; 101:301-9.

5. Waxler B, Dadabhoy Z, Stojiljkovic L. Primer of postoperative pruritus for anesthesiologists. Anesthesiology 2005; 103:168-178.

6. Sakakihara M, Imamachi N, Saito Y. Effects of epidural k-opioid receptor agonist on morphineinduced itch and antinociception in mice. Reg Anesth Pain Med 2016;41:69-74.

7. Miller JL, Hagemann TM. Use of pure opioid antagonists for management of opioid-induced pruritus. Am J Health Sys Pharm 2011;68:1419-25.

8. Yeh HM, Chen LK, Lin CJ et al. Prophylactic intravenous ondansetron reduces the incidence of epidural morphine-induced pruritus inpatients undergoing cesarean delivery. Anesth Analg 2000;91:172-175.

9. Horta ML, Ramos L, Goncalves ZR. The inhibition of epidural morphine-induced pruritus by epidural droperidol. Anesth Analg. 2000;90:638-641.

10. Szarvas S, Harmon D, Murphy D. Neuraxial opioid-induced pruritus: a review. J Clin Anesth 2003;15:234-239.

11. Bautista DM, Wilson SR, Hoon MA. Why we scratch an itch: the molecules cells and circuits of itch. Nat. Neurosci 2014;17:175-182. 
12. Barry DM, Munanairi A, Chen ZF. Spinal mechanisms of itch transmission. Neurosci Bull. 2018;34:156-164.

13. Papoiu ADP, Kraft RA, Coghill RC et al. Butorphanol suppression of histamine itch is mediated by nucleus accumbens and septal nuclei: a pharmaco-logical fMRI study. J Invest Dermatol 2015;135:560-568.

14. Kelly MC, Carabine UA, Mirakhur RK. Epidural diamorphine for analgesia after caesarean section. A dose finding study and assessment of side-effects. Anesthesia. 1998;53:231-237.

15. Viscusi ER, Gan TJ, Leslie JB, et al. Peripherally acting mu-opioid receptor antagonists and postoperative ileus: mechanisms of action and clinical applicability. Anesth Analg 2009; 108:181122.

16. Girgin NK, Gurbet A, Turker G, et al. Epidural morphine in anesthesia for combinations of low-dose epidural morphine and spinal bupivacaine. J Clin Anesth 2008;20:180-185.

17. Sarvela J, Halonen P, Soikkeli A, et al. A double-blinded, randomized comparison of epidural and epidural morphine for elective cesarean delivery. Anesth Analg 2002;95:436-440.

18. Colbert S, O'Hanlon DM, Galvin S, et al. The effect of rectal diclofenac on pruritus in patients receiving epidural morphine. Anesthesia 1999;54:948-952.

19. Ballantyne JC, Loach AB, Carr DB. Itching after epidural and spinal opiates. Pain 1998;33:149-160.

20. Jinks SL, Carstens E. Spinal NMDA receptor involvement in eapansion of dorsal horn neuronal receptive field area produced by intracutaneous histamine. J Neurophysiol 1998;79:1613-1618.

21. Chaney MA. Side effects of epidural and epidural opioids. Can J Anaesth. 1995;42:891-903.

\section{Figures}




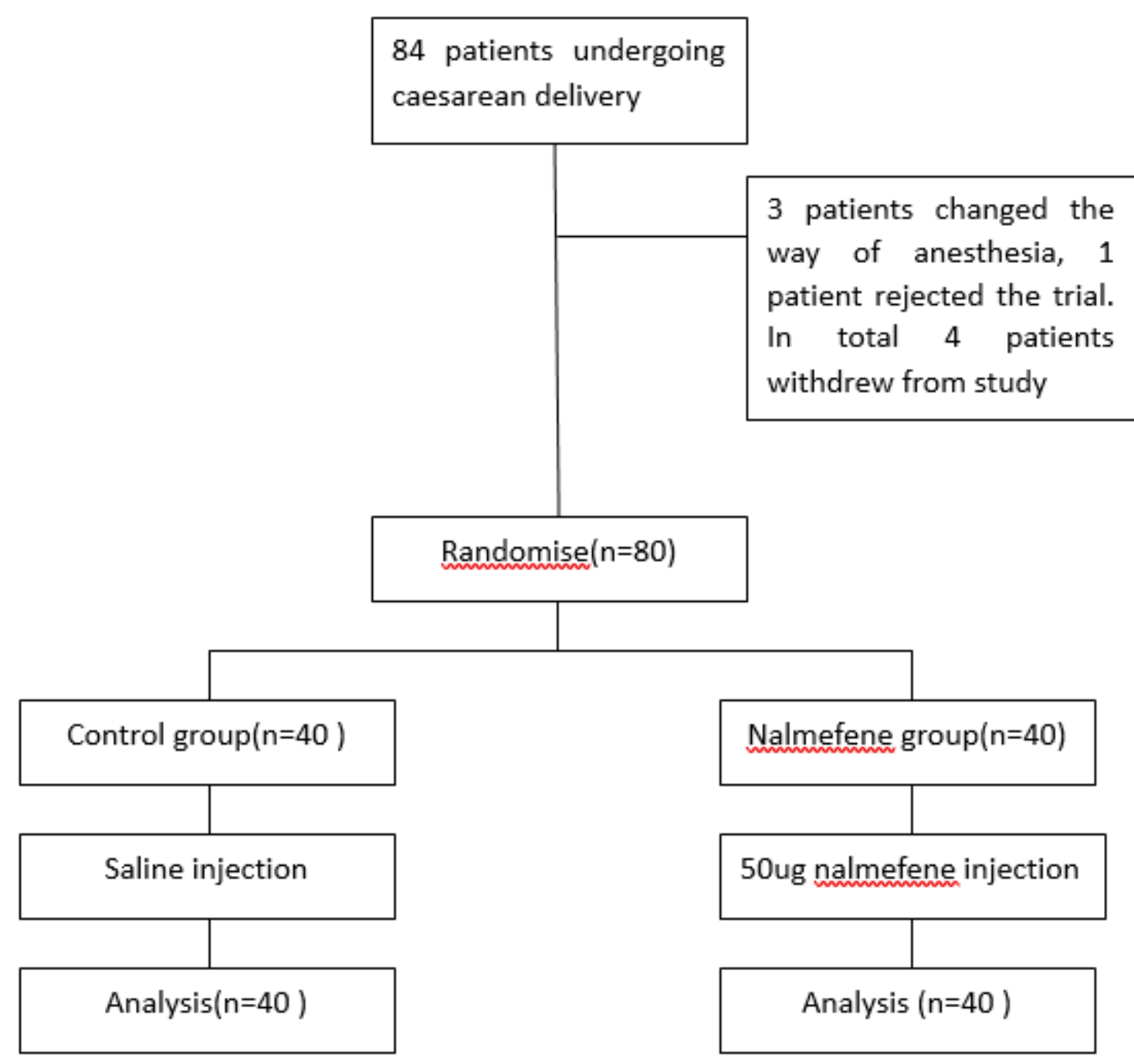

Figure 1

patient enrollment flow diagram. This illustrates the flow of all patients screened, excluded, and randomized

\section{Supplementary Files}

This is a list of supplementary files associated with this preprint. Click to download.

- CONSORTChecklist.doc 